\title{
Rate of Convergence of Tikhonov Method of Regularization for Constrained Linear Equations with Operators Having Closed Ranges
}

\author{
Milojica Jaćimović, Izedin Krnić, and Oleg Obradović \\ Department of Mathematics, University of Montenegro, 81000 Podgorica, Montenegro \\ Correspondence should be addressed to Milojica Jaćimović; milojica@jacimovic.me
}

Received 7 July 2013; Revised 11 September 2013; Accepted 13 September 2013

Academic Editor: Davide La Torre

Copyright (C) 2013 Milojica Jaćimović et al. This is an open access article distributed under the Creative Commons Attribution License, which permits unrestricted use, distribution, and reproduction in any medium, provided the original work is properly cited.

We derive the estimates of the rate of convergence of the Tikhonov method of regularization for a constrained operator linear equation. In case that the range of the corresponding operator is closed, the estimate is of the same order as the estimates for a linear equation without constraints.

\section{Introduction}

Let $H$ and $F$ be real Hilbert spaces, $A: H \mapsto F$-a linear bounded operator from $H$ to $F, f \in F-$ a fixed element, and $U \subseteq H$-a closed convex set. We will deal with the equation

$$
A u=f, \quad u \in U .
$$

Even when the constraint $u \in U$ is absent, this problem can be ill-posed; that is, it is possible that there is $\widetilde{u}$ which is far from the set of solution of $(1)$, such that $A \tilde{u} \approx f$. The ill-posedness of the equation in case of infinite-dimensional spaces $H$ and $F$ obviously comes from the fact that the range $R(A):=\{A u: u \in H\}$ of the operator $A$ is nonclosed. However, if the operator $A$ is known only approximately, then this equation can be ill-posed even in case of $R(A)=\overline{R(A)}$. In this case, in order to solve the given problem, one has to use methods of regularization, for example, the Tikhonov method of regularization (see $[1,2])$. Note that the presence of the constraints significantly complicates all issues concerning the ill-posedness.

Let us suppose that the set $U$ is given by

$$
U=\{u \in H: h(u) \leq 0\},
$$

where $h: H \mapsto R$ is a continuously differentiable convex function satisfying Lipschitz and Slater conditions:

$$
\begin{gathered}
(\exists L>0)(\forall u, v \in H) \quad\left\|h^{\prime}(u)-h^{\prime}(v)\right\| \leq L\|u-v\|, \\
\exists \bar{u} \in U: h(\bar{u})<0 .
\end{gathered}
$$

We also suppose that sets $U_{*}$ and $U_{\infty}$ of the solutions of (1), and the corresponding equation without constraint

$$
A u=f, \quad u \in H,
$$

are nonempty. In this case, the minimization problems

$$
\begin{gathered}
\|u\|^{2} \longmapsto \text { inf, } \quad u \in U_{*}, \\
\|u\|^{2} \longmapsto \text { inf, } \quad u \in U_{\infty}
\end{gathered}
$$

have unique solutions that will be denoted by $u_{*}$ and $u_{\infty}$ and called normal solutions of (1) and (5).

Let us present one prominent example of the equation with the previously mentioned constraint with infinitedimensional spaces $H$ and/or $F$.

Given that $T>0$ and matrices $C(\cdot)=\left(c_{i j}(\cdot)\right)_{m \times m}$, $D(\cdot)=\left(d_{i j}(\cdot)\right)_{m \times q}$ and $G(\cdot)=\left(g_{i j}(\cdot)\right)_{n \times m}$, whose elements $c_{i j}(\cdot), d_{i j}(\cdot)$, and $g_{i j}(\cdot)$ are piecewise continuous functions on 
$[0, T]$. Let us consider a system in the so-called state-variable (or state-space) form:

$$
\begin{gathered}
x^{\prime}(t)=C(t) x(t)+D(t) u(t), \quad t \in[0, T], x(0)=0, \\
y(t)=G(t) x(t),
\end{gathered}
$$

where $u=u(t), t \in[0, T]$ is a control from the space $L_{2}^{q}[0, T]$. This system of control is usually analyzed under some constraints on the control or on the trajectory. For example, the problems, "given $f \in R^{m}$, find a control

$$
\begin{aligned}
u & =u(\cdot) \in U \\
& =\left\{u \in L_{2}^{q}: \int_{0}^{T}\left|x^{\prime}(t, u(t))\right|^{2} d t \leq r^{2}\right\},
\end{aligned}
$$

such that $y(T)=y(T ; u)=f$ " is a problem of type (1), (2). Then, one has to solve the equation $A u=f$ on the set

$$
\begin{aligned}
U=\{u & \in H: h(u) \\
& \left.=\int_{0}^{T}\left|x^{\prime}(t, u(t))\right|^{2} d t-r^{2} \leq 0\right\},
\end{aligned}
$$

where

$$
A u=y(T ; u): H=L_{2}^{q}[0, T] \longmapsto F=R^{n} .
$$

For example, electrical networks with a finite number of interconnected resistors, capacitors, and inductors can be described in state-variable form (see [3]).

The problems of the type (1) in the literature are usually considered under the assumption that, instead of the exact operator $A$ and instead of the element $f$, one actually deals with their approximations $A_{\eta} \in \mathscr{L}(H, F), f_{\delta} \in F$, such that

$$
\left\|A-A_{\eta}\right\| \leq \eta, \quad\left\|f-f_{\delta}\right\| \leq \delta, \quad\left\|A_{\eta}\right\| \leq a,
$$

where $\eta>0, \delta>0$ are small positive real numbers and $a>0$.

As for the approximations of the normal solutions $u_{*}$ and $u_{\infty}$, one can use $[1,4]$ the so-called Tikhonov regularized approximate solutions $u_{\alpha}$ and $v_{\alpha}$, that is, the unique solutions of the regularized problems find $u_{\alpha} \in U$ such that

$$
\begin{gathered}
\left\langle A_{\eta}^{*} A_{\eta} u_{\alpha}-A_{\eta}^{*} f_{\delta}+\alpha u_{\alpha}, u-u_{\alpha}\right\rangle \geq 0, \quad \forall u \in U, \\
A_{\eta}^{*} A_{\eta} v_{\alpha}+\alpha v_{\alpha}=A_{\eta}^{*} f_{\delta} .
\end{gathered}
$$

Let us observe that the above variational inequality (13) is necessary and sufficient conditions for the minimization problem of the Tikhonov function

$$
T_{\alpha}(u)=\frac{1}{2}\left\|A_{\eta} u-f_{\delta}\right\|^{2}+\frac{\alpha}{2}\|u\|^{2}
$$

on the set $U$ or on the space $H$. The unique point $u_{\alpha}\left(=u_{\alpha(\delta, \eta)}\right)$ of the minimum of the function $T_{\alpha}$ on the set $U$ is said to be a regularized solution of (1).

The main aim of this paper is to establish whether there exists a choice of a parameter of regularization $\alpha=\alpha(\delta, \eta)$ which implies the convergence rate $O(\delta+\eta)$ of regularized solutions $u_{\alpha}=u_{\alpha(\delta, \eta)}$ to $u_{*}$. Namely, in [5], such convergence was proved in case of (5) without constraints and under the assumption that $\overline{R(A)}=R(A)$. Note also that even in case of $H=R^{2}$, the rate of convergence $u_{\alpha(\delta, \eta)} \rightarrow u_{*}$, depending on the boundary of the set $U$, can be arbitrarily slow (see [6]).

Consequently, even in case of $R(A)=\overline{R(A)}$, only if we require additional conditions on $h$ and $u_{*}$, we can eventually expect the convergence rate $O(\delta+\eta)$ of regularized solutions $u_{\alpha}$ to normal solution $u_{*}$. In [7], it was proved that if $u_{\infty}$ and projection of $h^{\prime}\left(u_{*}\right)$ belong to $R\left(A^{*}\right)$, then for $\alpha=O(\delta+\eta)$ yields $\left\|u_{\alpha}-u_{*}\right\|=O(\delta+\eta)^{1 / 2}$.

The estimates of the rate of convergence of regularized solutions $u_{\alpha}$ to the normal solution $u_{*}$ have been obtained in [8], under the conditions related to the smoothness of the boundary of the set $U$ and of the properties of the projection of $u_{*}$ onto linear subspace of codimension 1 , which is parallel to the tangential plane to the boundary of $U$.

Let us emphasize that the results of this paper are inspired by the results from [8]. However, our results related to the rate of convergence of regularized solutions were obtained under somewhat different general conditions and under the assumptions that, instead of the exact operator $A$, and instead of the element $f$, we only know their approximations $A_{\eta}$ and $f_{\delta}$.

The paper is organized as follows.

In Section 2, we described Tikhonov method of regularization adapted to the case when the set of constraints is given by (2). This constraint is taken into account by Lagrange multipliers. Lemma 1 was proved under the conditions of the sourcewise representativity type, that is, under the assumption that $u_{*}$ belongs to the projection of $\overline{R\left(A^{*}\right)}$ onto $U[2,6,8]$. In this section, we also present some results concerning the properties of the operators $A_{\eta}^{*} A_{\eta}$ and $A_{\eta} A_{\eta}^{*}$.

In Section 3, we suppose that the parameter of regularization $\alpha$ is chosen in such way that $\alpha=O(\delta+\eta)$. Under this assumption, we derive the estimates of the rate of convergence of Lagrange multipliers of regularized problems. The main result of the paper is contained in Theorem 11 which refers to the rate of convergence of regularized solutions.

\section{Constrained Tikhonov Regularization and Previous Results}

By applying the Kuhn-Tucker theorem to the minimization problem

$$
T_{\alpha}(u)=\frac{1}{2}\left\|A_{\eta} u-f_{\delta}\right\|^{2}+\frac{\alpha}{2}\|u\|^{2}, \quad h(u) \leq 0,
$$

we obtain that, for its unique solution $u_{\alpha}=u_{\alpha(\delta, \eta)}$, there is $\lambda_{\alpha} \geq 0$ such that

$$
\begin{gathered}
A_{\eta}^{*} A_{\eta} u_{\alpha}-A_{\eta}^{*} f_{\delta}+\alpha u_{\alpha}+\lambda_{\alpha} h^{\prime}\left(u_{\alpha}\right)=0, \\
\lambda_{\alpha} h\left(u_{\alpha}\right)=0 .
\end{gathered}
$$


Using the function $g_{\alpha}(t)=1 /(t+\alpha), t \geq 0$, (in what follows, we will use this notation) the first equality in (17) can be written as

$$
g_{\alpha}^{-1}\left(A_{\eta}^{*} A_{\eta}\right) u_{\alpha}-A_{\eta}^{*} f_{\delta}+\lambda_{\alpha} h^{\prime}\left(u_{\alpha}\right)=0,
$$

or as

$$
u_{\alpha}=v_{\alpha}-\lambda_{\alpha} g_{\alpha}\left(A_{\eta}^{*} A_{\eta}\right) h^{\prime}\left(u_{\alpha}\right),
$$

where $v_{\alpha}=g_{\alpha}\left(A_{\eta}^{*} A_{\eta}\right) A_{\eta}^{*} f_{\delta}$ is the point of the minimum of the Tikhonov function $T_{\alpha}$ on $H$.

In what follows, we assume that the parameter $\alpha$ is chosen such that $\alpha=O(\delta+\eta)$.

Note that if $h\left(v_{\alpha}\right) \leq 0$, then $u_{*}=u_{\infty}$, and for such $\alpha$, the corresponding estimates concerning the accuracy of the regularized solutions of the equation $A u=f, u \in H$, can be found, for example, in [5]. (Note that in [5], page 100, it was proved that if $R(A)=\overline{R(A)}$, then for $\alpha=O(\delta+\eta)$, the following estimate holds: $\left.\left\|u_{\infty}-v_{\alpha}\right\|=O(\delta+\eta)\right)$. For this reason, in what follows, we will suppose that $h\left(v_{\alpha}\right)>0$ for all sufficiently small $\delta$ and $\eta$. In this case, we have $\lambda_{\alpha} \neq 0$ and, consequently, $h\left(u_{\alpha}\right)=0$ and $h^{\prime}\left(u_{\alpha}\right) \neq 0$ for all such $\delta$ and $\eta$. Therefore, bearing in mind that $u_{\alpha} \rightarrow u_{*}$ as $\delta, \eta \rightarrow 0$, we conclude that $h\left(u_{*}\right)=0$. The Slater condition (4) then implies that $u_{*}$ is not a point of minimum of $h$, and, consequently, $h^{\prime}\left(u_{*}\right) \neq 0$.

Now, as a consequence of the variational inequality (13), taking into account the equality (17) and $\lambda_{\alpha}>0$, we obtain

$$
(\forall u \in U) \quad\left\langle h^{\prime}\left(u_{\alpha}\right), u-u_{\alpha}\right\rangle \leq 0 .
$$

From here, we have

$$
(\forall u \in U) \quad\left\langle h^{\prime}\left(u_{*}\right), u-u_{*}\right\rangle \leq 0 .
$$

Consequently,

$$
\left\langle h^{\prime}\left(u_{*}\right), u_{\alpha}-u_{*}\right\rangle \leq 0, \quad\left\langle h^{\prime}\left(u_{\alpha}\right), u_{*}-u_{\alpha}\right\rangle \leq 0 .
$$

Further estimates of the rate of convergence of the regularized solutions are based on the next property of the normal solution

$$
u_{\infty} \in \overline{R\left(A^{*}\right)}=\overline{R\left(A^{*} A\right)} \text {, }
$$

that for the operator with closed range becomes

$$
u_{\infty} \in R\left(A^{*} A\right) ; \quad \text { that is, } u_{\infty}=R\left(A^{*} A\right) w_{*}, \quad w_{*} \in H .
$$

As it was argued in [6] (see also [9]), this condition does not have the same meaning as it has in case of problem (6), because the normal solution $u_{*}$ of the corresponding problem without constraints does not satisfy the condition $u_{*} \in R\left(A^{*} A\right)$. However, it is easy to prove that

$$
u_{*} \in \overline{\pi_{U}\left(R\left(A^{*}\right)\right)} \text {, }
$$

where by $\pi_{X}$, we denote the operator of the metric projecting on the closed convex set $X \subseteq H$.
Lemma 1. $u_{*} \in \pi_{U}\left(\overline{\left.R\left(A^{*}\right)\right)}\right.$ if and only if there exists $\gamma_{*} \geq 0$ such that $u_{*}=u_{\infty}-\gamma_{*}\left(I-\pi \overline{R\left(A^{*} A\right)}\right) h^{\prime}\left(u_{*}\right)$.

Proof. For any $u_{*} \in \pi_{U}\left(\overline{R\left(A^{*}\right)}\right)$, there is $e_{*} \in \overline{R\left(A^{*}\right)}$ such that $u_{*}$ is a solution of the minimization problem

$$
\frac{1}{2}\left\|u-e_{*}\right\|^{2} \longrightarrow \text { inf, } \quad h(u) \leq 0 .
$$

According to the Kuhn-Tucker theorem, there exists a real number $\gamma_{*} \geq 0$ such that $u_{*}-e_{*}+\gamma_{*} h^{\prime}\left(u_{*}\right)=0$. From here, bearing in mind that $e_{*} \in \overline{R\left(A^{*}\right)}$, we obtain

$$
\left(I-\pi_{\overline{R\left(A^{*}\right)}}\right) u_{*}=-\gamma_{*}\left(I-\pi_{\overline{R\left(A^{*}\right)}}\right) h^{\prime}\left(u_{*}\right) .
$$

Consequently,

$$
\begin{aligned}
u_{*} & =u_{\infty}+\left(I-\pi \overline{R\left(A^{*}\right)}\right) u_{*} \\
& =u_{\infty}-\gamma_{*}\left(I-\pi \overline{R\left(A^{*}\right)}\right) h^{\prime}\left(u_{*}\right) .
\end{aligned}
$$

Now, let us suppose that there exists $\gamma_{*} \geq 0$ such that

$$
u_{*}=u_{\infty}-\gamma_{*}\left(I-\pi \overline{R\left(A^{*}\right)}\right) h^{\prime}\left(u_{*}\right) .
$$

This equality can be written in the form of

$$
u_{*}-\left(u_{\infty}-\gamma_{*} \pi \overline{R\left(A^{*}\right)} h^{\prime}\left(u_{*}\right)\right)=-\gamma_{*} h^{\prime}\left(u_{*}\right) .
$$

Multiplying both sides of this equality by $u-u_{*}$ and bearing in mind (21), it comes out that

$$
\begin{aligned}
\left\langle u_{*}\right. & \left.-\left(u_{\infty}+\gamma_{*} \pi \overline{R\left(A^{*}\right)} h^{\prime}\left(u_{*}\right)\right), u-u_{*}\right\rangle \\
& =-\gamma_{*}\left\langle h^{\prime}\left(u_{*}\right), u-u_{*}\right\rangle \geq 0, \quad u \in U .
\end{aligned}
$$

The last equality means that $u_{*}=\pi_{U}\left(u_{\infty}+\gamma_{*} \pi \overline{R\left(A^{*}\right)} h^{\prime}\left(u_{*}\right)\right)$. Having in mind that $u_{\infty}+\gamma_{*} \pi \overline{R\left(A^{*}\right)} h^{\prime}\left(u_{*}\right) \in \overline{R\left(A^{*}\right)}$, it follows that $u_{*} \in \pi_{U}\left(\overline{R\left(A^{*}\right)}\right)$.

As an immediate consequence of this lemma, we have that if the range of the operator $A: H \rightarrow F$ is closed subspace of $F$, then $u_{*} \in \pi_{U}\left(R\left(A^{*}\right)\right)$ if and only if there is $\gamma_{*} \geq 0$ such that $u_{*}=u_{\infty}-\gamma_{*}\left(I-\pi_{R\left(A^{*}\right)}\right) h^{\prime}\left(u_{*}\right)$.

Finally, let us remark that it is easy to construct an example of convex set $U \subseteq R^{2}$ and operator $A: R^{2} \mapsto R^{2}$ such that $u_{*} \notin \pi_{U}\left(R\left(A^{*}\right)\right)$.

Further, we will continue with three lemmas related to properties of the operators $A_{\eta}^{*} A_{\eta}$ and $A_{\eta} A_{\eta}^{*}$, whose proofs can be found in [5], pages 99, 153, 154, and 156 .

Lemma 2. If $\left\|A-A_{\eta}\right\| \leq \eta$, then

$$
\left(g_{\alpha}\left(A_{\eta}^{*} A_{\eta}\right) A_{\eta}^{*} A_{\eta}-I\right) h \longrightarrow 0 \quad \text { as } \alpha \longrightarrow 0, \eta \longrightarrow 0
$$

for all $h \in \overline{R\left(A^{*}\right)}$. 
Lemma 3. (a) The range of the bounded linear operator $A$ : $H \rightarrow F$ is closed subspace of $H$ if and only if

$$
m_{A}:=\inf \{\|A u\|:\|u\|=1, u \perp \operatorname{Ker} A\}>0 .
$$

(b) If the bounded linear operators $A: H \mapsto F$ and $A_{\eta}$ : $H \mapsto F$ satisfy the condition

$$
m_{A}>0, \quad\left\|A-A_{\eta}\right\| \leq \eta, \quad 0<2 \eta<m_{A},
$$

then $\sigma\left(A_{\eta}^{*} A_{\eta}\right), \sigma\left(A_{\eta} A_{\eta}^{*}\right) \subseteq\left[0, \eta^{2}\right] \bigcup\left[\left(m_{A}-\eta\right)^{2},\left\|A_{\eta}\right\|^{2}\right]$, where $\sigma\left(A_{\eta}^{*} A_{\eta}\right)$ and $\sigma\left(A_{\eta} A_{\eta}^{*}\right)$ are the spectrums of the operators $A_{\eta}^{*} A_{\eta}$, and $A_{\eta} A_{\eta}^{*}$.

By $\pi_{\eta}$, we will denote the operator of orthogonal projecting of the space $H$ on invariant subspace of the operator $A_{\eta}^{*} A_{\eta}$ that corresponds to the part of the spectrum belonging to $\left[\left(m_{A}-\eta\right)^{2},\left\|A_{\eta}\right\|^{2}\right]$. Then, $I-\pi_{\eta}$ is the operator of orthogonal projecting of the space $H$ on invariant subspace of the operator $A_{\eta}^{*} A_{\eta}$ that corresponds to the part of the spectrum belonging to $\left[0, \eta^{2}\right]$.

Lemma 4. If the conditions (34) are satisfied, then,

$$
\begin{aligned}
& \left\|\pi_{R\left(A^{*}\right)}-\pi_{\eta}\right\| \leq \frac{\eta}{m_{A}-\eta}, \\
& \left\|\pi_{\eta}\left(I-\pi_{\overline{R\left(A^{*}\right)}}\right)\right\| \leq \frac{\eta}{m_{A}-\eta}, \\
& \left\|A_{\eta}\left(I-\pi_{\eta}\right)\right\| \leq \eta
\end{aligned}
$$

As the consequences of the previous lemmas, it is easy to prove the following estimates.

Lemma 5. Let the conditions (34) be satisfied. Then

$$
\left\|\pi_{\eta} g_{\alpha}\left(A_{\eta}^{*} A_{\eta}\right)\right\| \leq \frac{1}{\left(m_{A}-\eta\right)^{2}} .
$$

If, additionally, the parameter $\alpha$ of regularization is chosen such that $\alpha=O(\delta+\eta)$, then the following estimates hold for all $x$ :

$$
\begin{gathered}
\left\|g_{\alpha}\left(A_{\eta}^{*} A_{\eta}\right) A_{\eta}^{*}\right\| \leq \max \left\{1 ; \frac{1}{m_{A}-\eta}\right\} \\
\left\|\left(I-\alpha g_{\alpha}\left(A_{\eta}^{*} A_{\eta}\right)\right)\left(I-\pi_{\eta}\right) x\right\| \\
\leq \max \left\{1 ; \frac{1}{m_{A}-\eta}\right\}\left\|\left(I-\pi_{\eta}\right) x\right\| \eta, \\
\left\|\alpha g_{\alpha}\left(A_{\eta}^{*} A_{\eta}\right) x-\left(I-\pi_{\eta}\right) x\right\| \\
\leq\left(\frac{1}{\left(m_{A}-\eta\right)^{2}}+\max \left\{1 ; \frac{1}{m_{A}-\eta}\right\}\right)\|x\|(\delta+\eta) .
\end{gathered}
$$

The last result that we will cite in this section concerns the rate of convergence of the Tikhonov regularization of the equation $A u=f, u \in H$, and its proof can be found in [4] and also in [5].
Theorem 6. If the conditions (12) are satisfied and the parameter $\alpha=\alpha(\delta, \eta)$ is chosen such that $\alpha(\delta, \eta) \rightarrow 0$ and $\sqrt{\delta+\eta} / \alpha \rightarrow 0$ as $\delta \rightarrow 0, \eta \rightarrow 0$, then the regularized solutions $v_{\alpha(\delta, \eta)}$ of (14) converge to the normal solution $u_{\infty}$ of (5) as $\delta \rightarrow 0, \eta \rightarrow 0$.

If, additionally, the range of the operator $A$ is closed, then for $\alpha=O(\delta+\eta)$, the following estimate holds:

$$
\left\|v_{\alpha}-u_{\infty}\right\|=O(\delta+\eta) \text {. }
$$

\section{Convergence and Rate of Convergence}

In order to derive an estimate for the solutions of the equation $A u=f, u \in U \subseteq H$, we will begin this section with lemmas concerning the properties of Lagrange multipliers $\lambda_{\alpha}$ for problem (17), that will be used in the proof of the estimates of the rate of convergence. Note that throughout the section we will suppose that the parameter of the regularization $\alpha$ is chosen such that $\alpha=O(\delta+\eta)$.

Lemma 7. If the conditions (34), (12) are satisfied and (I$\pi) h^{\prime}\left(u_{*}\right) \neq 0$, then for $\alpha=O(\delta+\eta)$, the following estimate is true:

$$
\left|\frac{\lambda_{\alpha}}{\alpha}-\gamma_{*}\right|=O\left(\delta+\eta+\left\|u_{*}-u_{\alpha}\right\|\right),
$$

where $\lambda_{\alpha}$ and $\gamma_{*}$ are Lagrange multipliers from (17) and Lemma 2.

Proof. By (39) and Lemma 4, from the equality

$$
\begin{aligned}
\alpha g_{\alpha}\left(A_{\eta}^{*} A_{\eta}\right) h^{\prime}\left(u_{*}\right)-\left(I-\pi_{\overline{R\left(A^{*}\right)}}\right) h^{\prime}\left(u_{*}\right) \\
=\alpha g_{\alpha}\left(A_{\eta}^{*} A_{\eta}\right) h^{\prime}\left(u_{*}\right)-\left(I-\pi_{\eta}\right) h^{\prime}\left(u_{*}\right) \\
+\left(\pi_{\overline{R\left(A^{*}\right)}}-\pi_{\eta}\right) h^{\prime}\left(u_{*}\right),
\end{aligned}
$$

we get that

$$
\begin{gathered}
\alpha g_{\alpha}\left(A_{\eta}^{*} A_{\eta}\right) h^{\prime}\left(u_{*}\right) \longrightarrow\left(I-\pi \overline{R\left(A^{*}\right)}\right) h^{\prime}\left(u_{*}\right) \\
\text { as } \delta, \eta \longrightarrow 0, \alpha=O(\delta+\eta)
\end{gathered}
$$

Furthermore, since $u_{\alpha} \rightarrow u_{*}, h^{\prime}\left(u_{\alpha}\right) \rightarrow h^{\prime}\left(u_{*}\right)$, we have

$$
\begin{gathered}
\left\langle\alpha g_{\alpha}\left(A_{\eta}^{*} A_{\eta}\right) h^{\prime}\left(u_{*}\right), h^{\prime}\left(u_{\alpha}\right)\right\rangle \\
\longrightarrow\left\|\left(I-\pi_{\overline{R\left(A^{*}\right)}}\right) h^{\prime}\left(u_{*}\right)\right\|^{2} \neq 0 \\
\text { as } \alpha=O(\delta+\eta) \longrightarrow 0 .
\end{gathered}
$$

Consequently,

$$
\left\langle\alpha g_{\alpha}\left(A_{\eta}^{*} A_{\eta}\right) h^{\prime}\left(u_{*}\right), h^{\prime}\left(u_{\alpha}\right)\right\rangle \neq 0
$$

for all sufficiently small $\delta$ and $\eta$. Multiplying both sides of (19) by $h^{\prime}\left(u_{*}\right)$ and taking into consideration the last inequality, we obtain

$$
\frac{\lambda_{\alpha}}{\alpha}=\frac{\left\langle h^{\prime}\left(u_{*}\right), v_{\alpha}-u_{\alpha}\right\rangle}{\left\langle\alpha g_{\alpha}\left(A_{\eta}^{*} A_{\eta}\right) h^{\prime}\left(u_{*}\right), h^{\prime}\left(u_{\alpha}\right)\right\rangle} .
$$


Now, multiplying the equality $u_{*}=u_{\infty}-\gamma_{*}\left(I-\pi \overline{R\left(A^{*}\right)}\right) h^{\prime}\left(u_{*}\right)$ (see Lemma 1) by $\left(I-\pi \overline{R\left(A^{*}\right)}\right) h^{\prime}\left(u_{*}\right)$ and taking into account that $u_{\infty}-u_{*} \in \operatorname{Ker} A$, we find that

$$
\gamma_{*}=\frac{\left\langle h^{\prime}\left(u_{*}\right), u_{\infty}-u_{*}\right\rangle}{\left\|\left(I-\pi \overline{R\left(A^{*}\right)}\right) h^{\prime}\left(u_{*}\right)\right\|^{2}} .
$$

In this way, we obtain the equality

$$
\begin{aligned}
& \gamma_{*}-\frac{\lambda_{\alpha}}{\alpha} \\
& =\frac{\left\langle h^{\prime}\left(u_{*}\right), u_{\infty}-v_{\alpha}\right\rangle+\left\langle h^{\prime}\left(u_{*}\right), u_{\alpha}-u_{*}\right\rangle}{\left\|\left(I-\pi_{\overline{R\left(A^{*}\right)}}\right) h^{\prime}\left(u_{*}\right)\right\|^{2}} \\
& +\frac{\left\langle h^{\prime}\left(u_{*}\right), v_{\alpha}-u_{\alpha}\right\rangle}{\left\|\left(I-\pi_{\overline{R\left(A^{*}\right)}}\right) h^{\prime}\left(u_{*}\right)\right\|^{2}\left\langle\alpha g_{\alpha}\left(A_{\eta}^{*} A_{\eta}\right) h^{\prime}\left(u_{*}\right), h^{\prime}\left(u_{\alpha}\right)\right\rangle} \\
& \quad \times\left[\left\langle\alpha g_{\alpha}\left(A_{\eta}^{*} A_{\eta}\right) h^{\prime}\left(u_{*}\right), h^{\prime}\left(u_{\alpha}\right)\right\rangle-\left\|\left(I-\pi_{\overline{R\left(A^{*}\right)}}\right) u_{*}\right\|^{2}\right] .
\end{aligned}
$$

Let us observe that

$$
\begin{aligned}
\left\langle\alpha g_{\alpha}\left(A_{\eta}^{*} A_{\eta}\right) h^{\prime}\left(u_{*}\right), h^{\prime}\left(u_{\alpha}\right)\right\rangle & -\left\|\left(I-\pi_{\overline{R\left(A^{*}\right)}}\right) u_{*}\right\|^{2} \\
= & \left\langle\alpha g_{\alpha}\left(A_{\eta}^{*} A_{\eta}\right) h^{\prime}\left(u_{*}\right), h^{\prime}\left(u_{\alpha}\right)-h^{\prime}\left(u_{*}\right)\right\rangle \\
& +\left\langle\alpha g_{\alpha}\left(A_{\eta}^{*} A_{\eta}\right) g^{\prime}\left(u_{*}\right)\right. \\
& \left.-\left(I-\pi_{\eta}\right) h^{\prime}\left(u_{*}\right), g^{\prime}\left(u_{*}\right)\right\rangle \\
+ & \left\langle\left(\pi_{\overline{R\left(A^{*}\right)}}-\pi_{\eta}\right) h^{\prime}\left(u_{*}\right), h^{\prime}\left(u_{*}\right)\right\rangle .
\end{aligned}
$$

Now, having in mind condition (3), estimate (39), Lemma 4 , and Theorem 6, we get

$$
\left|\gamma_{*}-\frac{\lambda_{\alpha}}{\alpha}\right|=O\left(\delta+\eta+\left\|u_{*}-u_{\alpha}\right\|\right)
$$

In the following theorem, we will present one result concerning the estimates of the discrepancies $\left\|A u_{\alpha}-f\right\|$ and $\left\|A_{\eta} u_{\alpha}-f_{\delta}\right\|$.

Theorem 8. If the conditions (34) and (12) are satisfied and $u_{*} \in \pi_{U}\left(R\left(A^{*}\right)\right)$, then for $\alpha=O(\delta+\eta)$, the following estimates hold:

$$
\begin{gathered}
\left\|A\left(u_{*}-u_{\alpha}\right)\right\|=O(\delta+\eta), \quad\left\|A_{\eta}\left(u_{*}-u_{\alpha}\right)\right\|=O(\delta+\eta), \\
\left\|\frac{A_{\eta} u_{\alpha}-f_{\delta}}{\alpha}\right\|=O(1) .
\end{gathered}
$$

Proof. First, let us consider the case when $\left(I-\pi_{\overline{R\left(A^{*}\right)}}\right) h^{\prime}\left(u_{*}\right)=$ 0 . Then, based on (19) and Lemma 1 , we have that $u_{*}=u_{\infty}$ and

$$
\begin{aligned}
u_{*}-u_{\alpha}= & u_{\infty}-v_{\alpha} \\
& +\lambda_{\alpha} g_{\alpha}\left(A_{\eta}^{*} A_{\eta}\right) h^{\prime}\left(u_{\alpha}\right) .
\end{aligned}
$$

Multiplying both sides of this equality by $g_{\alpha}^{-1}\left(A_{\eta}^{*} A_{\eta}\right)\left(u_{*}-u_{\alpha}\right)$ and taking into account (22), we obtain

$$
\begin{aligned}
& \left\langle g_{\alpha}^{-1}\left(A_{\eta}^{*} A_{\eta}\right)\left(u_{*}-u_{\alpha}\right), u_{*}-u_{\alpha}\right\rangle \\
& \quad \leq\left\langle g_{\alpha}^{-1}\left(A_{\eta}^{*} A_{\eta}\right)\left(u_{\infty}-v_{\alpha}\right), u_{*}-u_{\alpha}\right\rangle .
\end{aligned}
$$

The last inequality can be written in the form

$$
\begin{aligned}
& \left\|A_{\eta}\left(u_{*}-u_{\alpha}\right)\right\|^{2}+\alpha\left\|u_{*}-u_{\alpha}\right\|^{2} \\
& \quad \leq\left\|A_{\eta}\left(u_{\infty}-v_{\alpha}\right)\right\|^{2}+\alpha\left\|u_{\infty}-v_{\alpha}\right\|^{2} .
\end{aligned}
$$

In this case, from here and from Theorem 6 , it follows that

$$
\begin{gathered}
\left\|A_{\eta}\left(u_{*}-u_{\alpha}\right)\right\|=O(\delta+\eta), \\
\left\|A\left(u_{*}-u_{\alpha}\right)\right\| \leq \\
\quad\left\|\left(A-A_{\eta}\right)\left(u_{*}-u_{\alpha}\right)\right\| \\
+\left\|A_{\eta}\left(u_{*}-u_{\alpha}\right)\right\|=O(\delta+\eta) .
\end{gathered}
$$

Now, let us consider the case of $\left(I-\pi_{\overline{R\left(A^{*}\right)}}\right) h^{\prime}\left(u_{*}\right) \neq 0$. Then, we have that $u_{*}=u_{\infty}-\gamma_{*}\left(I-\pi \overline{R\left(A^{*}\right)}\right) h^{\prime}\left(u_{*}\right)$; that is

$$
\begin{aligned}
u_{*}-u_{\alpha}= & u_{\infty}-v_{\alpha} \\
& +\lambda_{\alpha} g_{\alpha}\left(A_{\eta}^{*} A_{\eta}\right) h^{\prime}\left(u_{\alpha}\right) \\
& -\gamma_{*}\left(I-\pi_{\overline{R\left(A^{*}\right)}}\right) h^{\prime}\left(u_{*}\right),
\end{aligned}
$$

from where we obtain that

$$
\begin{aligned}
A_{\eta}\left(u_{*}-u_{\alpha}\right)= & A_{\eta}\left(u_{\infty}-v_{\alpha}\right) \\
& +\alpha \frac{\lambda_{\alpha}}{\alpha} A_{\eta} g_{\alpha}\left(A_{\eta}^{*} A_{\eta}\right) h^{\prime}\left(u_{\alpha}\right) \\
& -\gamma_{*}\left(A_{\eta}-A\right) \\
& \times\left(I-\pi_{\overline{R\left(A^{*}\right)}}\right) h^{\prime}\left(u_{*}\right) .
\end{aligned}
$$

From here, by Theorem 6, Lemma 5 and having in mind (37) and condition (12), we obtain the estimate

$$
\begin{gathered}
\left\|A_{\eta}\left(u_{*}-u_{\alpha}\right)\right\|=O(\delta+\eta), \\
\left\|A\left(u_{*}-u_{\alpha}\right)\right\|=O(\delta+\eta) .
\end{gathered}
$$

The third estimate in the theorem is an immediate consequence of (12) and the equality

$$
\begin{aligned}
A_{\eta} u_{\alpha}-f_{\delta}= & A_{\eta}\left(u_{\alpha}-u_{*}\right) \\
& +\left(A_{\eta}-A\right) u_{*}+f-f_{\delta} .
\end{aligned}
$$


Before presenting the proof of the main result, we will prove two lemmas: the first regarding the convergence of Lagrange multipliers $\lambda_{\alpha}$ from equality (17) and the second concering the projection of $u_{*}-u_{\alpha}$ on the subspace $\overline{R\left(A^{*} A\right)}$.

Lemma 9. Suppose the conditions (34) and (12) are satisfied and $u_{*} \in \pi_{U}\left(R\left(A^{*}\right),\left(I-\pi_{\overline{R\left(A^{*}\right)}}\right) h^{\prime}\left(u_{*}\right)=0\right.$. Then, for $\alpha=$ $\mathrm{O}(\delta+\eta)$ one has

$$
\frac{\lambda_{\alpha}}{\alpha}=O(1)
$$

Proof. From $\left(I-\pi_{\overline{R\left(A^{*}\right)}}\right) h^{\prime}\left(u_{*}\right)=0$, it follows that $u_{*}=u_{\infty}$ and

$$
h^{\prime}\left(u_{*}\right)=\pi \overline{R\left(A^{*}\right)} h^{\prime}\left(u_{*}\right) \in R\left(A^{*} A\right) .
$$

Then, there is $e \in R\left(A^{*} A\right)$ such that $h^{\prime}\left(u_{*}\right)=A^{*} A e$ and consequently

$$
\begin{aligned}
g_{\alpha}\left(A_{\eta}^{*} A_{\eta}\right) h^{\prime}\left(u_{*}\right)= & g_{\alpha}\left(A_{\eta}^{*} A_{\eta}\right) A^{*} A e \\
= & g_{\alpha}\left(A_{\eta}^{*} A_{\eta}\right)\left(A^{*} A-A_{\eta}^{*} A_{\eta}\right) e \\
& +\left(g_{\alpha}\left(A_{\eta}^{*} A_{\eta}\right) A_{\eta}^{*} A_{\eta}-I\right) h+h .
\end{aligned}
$$

Adding and subtracting $\left(I-\pi_{\eta}\right)\left(\left(A^{*} A-A_{\eta}^{*} A_{\eta}\right) / \alpha\right) e$ on the right side of this equality and then multiplying both sides by $h^{\prime}\left(u_{\alpha}\right)$, we obtain

$$
\begin{aligned}
\left\langle g_{\alpha}\left(A_{\eta}^{*} A_{\eta}\right) h^{\prime}\left(u_{*}\right), h^{\prime}\left(u_{\alpha}\right)\right\rangle & \\
= & \left\langle\left(\alpha g_{\alpha}\left(A_{\eta}^{*} A_{\eta}\right) \frac{A^{*} A-A_{\eta}^{*} A_{\eta}}{\alpha} e\right.\right. \\
& \left.\left.-\left(I-\pi_{\eta}\right) \frac{A^{*} A-A_{\eta}^{*} A_{\eta}}{\alpha} e\right), h^{\prime}\left(u_{\alpha}\right)\right\rangle \\
+ & \left\langle\frac{A^{*} A-A_{\eta}^{*} A_{\eta}}{\alpha} e,\left(I-\pi_{\overline{R\left(A^{*}\right)}}\right) h^{\prime}\left(u_{\alpha}\right)\right\rangle \\
+ & \left\langle\left(g_{\alpha}\left(A_{\eta}^{*} A_{\eta}\right) A_{\eta}^{*} A_{\eta}-I\right) e, h^{\prime}\left(u_{\alpha}\right)\right\rangle \\
+ & \left\langle e, h^{\prime}\left(u_{\alpha}\right)\right\rangle .
\end{aligned}
$$

Observe that $\left\|\left(I-\pi_{\overline{R\left(A^{*}\right)}}\right)\left(\left(A^{*} A-A_{\eta}^{*} A_{\eta}\right) / \alpha\right) e\right\|$ remains bounded when $\delta \rightarrow 0, \eta \rightarrow 0$ and $\alpha=O(\delta+\eta)$. Now, using (37), we have that

$$
\begin{aligned}
\| \alpha g_{\alpha}\left(A_{\eta}^{*} A_{\eta}\right) \frac{A^{*} A-A_{\eta}^{*} A_{\eta}}{\alpha} e \\
\quad-\left(I-\pi_{\eta}\right) \frac{A^{*} A-A_{\eta}^{*} A_{\eta}}{\alpha} e \| \\
=O\left(\left\|\left(I-\pi_{\eta}\right) \frac{A^{*} A-A_{\eta}^{*} A_{\eta}}{\alpha} e\right\|(\delta+\eta)\right) \rightarrow 0 .
\end{aligned}
$$

Besides, $\left(g_{\alpha}\left(A_{\eta}^{*} A_{\eta}\right) A_{\eta}^{*} A_{\eta}-I\right) e \rightarrow 0($ Lemma 2$)$ and

$$
\begin{array}{r}
\left(I-\pi_{\eta}\right) h^{\prime}\left(u_{\alpha}\right) \longrightarrow\left(I-\pi_{\overline{R\left(A^{*}\right)}}\right) h^{\prime}\left(u_{*}\right) \longrightarrow 0 \\
\text { as } \alpha=O(\delta+\eta) \longrightarrow 0 .
\end{array}
$$

Now, from (63), it follows that

$$
\begin{aligned}
\lim _{\alpha \rightarrow 0} & \left\langle g_{\alpha}\left(A_{\eta}^{*} A_{\eta}\right) h^{\prime}\left(u_{*}\right), h^{\prime}\left(u_{\alpha}\right)\right\rangle \\
& =\left\langle e, h^{\prime}\left(u_{*}\right)\right\rangle \\
& =\left\langle e, A^{*} A e\right\rangle \\
& =\|A e\|^{2} \\
& \neq 0 .
\end{aligned}
$$

It means that $\left\langle g_{\alpha}\left(A_{\eta}^{*} A_{\eta}\right) h^{\prime}\left(u_{*}\right), h^{\prime}\left(u_{\alpha}\right)\right\rangle \neq 0$ for all sufficiently small $\delta$ and $\eta$. Multiplying (19) by $h^{\prime}\left(u_{*}\right)$ and taking into account the last inequality, we will obtain

$$
\frac{\lambda_{\alpha}}{\alpha}=\frac{1}{\alpha} \frac{\left\langle h^{\prime}\left(u_{*}\right), v_{\alpha}-u_{\alpha}\right\rangle}{\left\langle g_{\alpha}\left(A_{\eta}^{*} A_{\eta}\right) h^{\prime}\left(u_{*}\right), h^{\prime}\left(u_{\alpha}\right)\right\rangle} .
$$

If we replace $h^{\prime}\left(u_{*}\right)$ and $u_{*}$ in the previous equality by $A^{*} A e$ and $u_{\infty}$, it becomes

$$
\frac{\lambda_{\alpha}}{\alpha}=\frac{1}{\alpha} \frac{\left\langle A e, A\left(v_{\alpha}-u_{\infty}\right)+A\left(u_{*}-u_{\alpha}\right)\right\rangle}{\left\langle g_{\alpha}\left(A_{\eta}^{*} A_{\eta}\right) h^{\prime}\left(u_{*}\right), h^{\prime}\left(u_{\alpha}\right)\right\rangle} .
$$

Based on Theorem 8, it follows the boundness of $\| A\left(v_{\alpha}-\right.$ $\left.u_{\infty}\right) \| / \alpha$ and $\left\|A\left(u_{*}-u_{\alpha}\right)\right\| / \alpha$ as $\alpha=O(\delta+\eta) \rightarrow 0$. Now, it is clear that $\lambda_{\alpha} / \alpha$ also remains bounded as $\alpha=O(\delta+\eta)$.

Lemma 10. Suppose the conditions (34) and (12) are satisfied. If $\alpha=O(\delta+\eta)$, then $\left\|\pi_{\overline{R\left(A^{*}\right)}}\left(u_{*}-u_{\alpha}\right)\right\|=O(\delta+\alpha)$.

Proof. From

$$
\begin{aligned}
u_{*}-u_{\alpha}= & u_{\infty}-v_{\alpha} \\
& +\lambda_{\alpha} g_{\alpha}\left(A_{\eta}^{*} A_{\eta}\right) h^{\prime}\left(u_{\alpha}\right) \\
& -\gamma_{*}\left(I-\pi \overline{R\left(A^{*}\right)}\right) h^{\prime}\left(u_{*}\right),
\end{aligned}
$$

we have that

$$
\begin{aligned}
\pi_{\eta}\left(u_{*}-u_{\alpha}\right)= & \pi_{\eta}\left(u_{\infty}-w_{\alpha}\right) \\
& +\frac{\lambda_{\alpha}}{\alpha} \alpha g_{\alpha}\left(A_{\eta}^{*} A_{\eta}\right) \pi_{\eta} h^{\prime}\left(u_{\alpha}\right) \\
& -\gamma_{*} \pi_{\eta}\left(I-\pi_{\overline{R\left(A^{*}\right)}}\right) h^{\prime}\left(u_{*}\right) .
\end{aligned}
$$


Consequently,

$$
\begin{aligned}
\pi_{\overline{R\left(A^{*}\right)}}\left(u_{*}-u_{\alpha}\right)= & \left(\pi_{\overline{R\left(A^{*}\right)}}-\pi_{\eta}\right) \\
& \times\left(u_{*}-u_{\alpha}\right)+\pi_{\eta}\left(u_{\infty}-v_{\alpha}\right) \\
& +\frac{\lambda_{\alpha}}{\alpha} \alpha g_{\alpha}\left(A_{\eta}^{*} A_{\eta}\right) \pi_{\eta} h^{\prime}\left(u_{\alpha}\right) \\
& -\gamma_{*} \pi_{\eta}\left(I-\pi_{\overline{R\left(A^{*}\right)}}\right) h^{\prime}\left(u_{*}\right) .
\end{aligned}
$$

The value $\lambda_{\alpha} / \alpha$ remains bounded as the parameter $\alpha=O(\delta+$ $\eta)$ converges to zero, in case of $\left(I-\pi \overline{R\left(A^{*}\right)}\right) h^{\prime}\left(u_{*}\right)=0$, as well as in case of $\left(I-\pi \overline{R\left(A^{*}\right)}\right) h^{\prime}\left(u_{*}\right) \neq 0$. Now, for $\alpha=O(\delta+\eta)$, the estimate

$$
\left\|\pi \overline{R\left(A^{*}\right)}\left(u_{*}-u_{\alpha}\right)\right\|=O(\delta+\eta)
$$

follows from

$$
\begin{aligned}
& \left\|\pi_{\eta}-\pi_{\overline{R\left(A^{*}\right)}}\right\|=O(\eta), \\
& \left\|u_{\infty}-v_{\alpha}\right\|=O(\delta+\eta), \\
& \left\|\pi_{\eta}\left(I-\pi \overline{R\left(A^{*}\right)}\right)\right\|=O(\eta), \\
& \left\|\pi_{\eta} g_{\alpha}\left(A_{\eta}^{*} A_{\eta}\right)\right\| \leq \frac{1}{\left(m_{A}-\eta\right)^{2}} .
\end{aligned}
$$

The following theorem is the main result of the paper.

Theorem 11. Suppose the conditions (12) and (34) are satisfied and $u_{*} \in \pi_{R\left(A^{*}\right)}$ and $\alpha=O(\delta+\eta)$. Then,

$$
\left\|u_{*}-u_{\alpha}\right\|=O(\delta+\eta) .
$$

Proof. From (17), we obtain the following equality:

$$
\begin{aligned}
\left(I-\pi_{\overline{R\left(A^{*}\right)}}\right) u_{\alpha}= & \frac{1}{\alpha}\left(I-\pi_{\overline{R\left(A^{*}\right)}}\right) \\
& \times\left(A^{*}-A_{\eta}^{*}\right)\left(f_{\delta}-A_{\eta} u_{\alpha}\right) \\
& -\frac{\lambda_{\alpha}}{\alpha}\left(I-\pi \overline{R\left(A^{*}\right)}\right) h^{\prime}\left(u_{\alpha}\right) .
\end{aligned}
$$

From here, taking into account the equality $u_{*}=u_{\infty}-\gamma_{*}(I-$ $\left.\pi \frac{}{R\left(A^{*}\right)}\right) h^{\prime}\left(u_{*}\right)$, we have

$$
\begin{aligned}
& u_{*}-u_{\alpha}=\pi \frac{}{R\left(A^{*}\right)}\left(u_{*}-u_{\alpha}\right) \\
& +\left(I-\pi_{\overline{R\left(A^{*}\right)}}\right) u_{*}-\left(I-\pi_{\overline{R\left(A^{*}\right)}}\right) u_{\alpha} \\
& =\pi \frac{}{R\left(A^{*}\right)}\left(u_{*}-u_{\alpha}\right) \\
& +\frac{\lambda_{\alpha}}{\alpha}\left(I-\pi_{\overline{R\left(A^{*}\right)}}\right) h^{\prime}\left(u_{\alpha}\right) \\
& -\gamma_{*}\left(I-\pi_{\overline{R\left(A^{*}\right)}}\right) h^{\prime}\left(u_{*}\right) \\
& +\frac{1}{\alpha}\left(I-\pi \overline{R\left(A^{*}\right)}\right) \\
& \times\left(A^{*}-A_{\eta}^{*}\right)\left(A_{\eta} u_{\alpha}-f_{\delta}\right) .
\end{aligned}
$$

Multiplying both sides of this equality by $u_{*}-u_{\alpha}$ and taking into account (13), we obtain

$$
\begin{aligned}
\left\|u_{*}-u_{\alpha}\right\|^{2} & \\
= & \left\langle\pi \frac{}{R\left(A^{*}\right)}\left(u_{*}-u_{\alpha}\right)+\left(I-\pi_{\overline{R\left(A^{*}\right)}}\right)\right. \\
& \left.\quad \times\left(A^{*}-A_{\eta}^{*}\right) \frac{A_{\eta} u_{\alpha}-f_{\delta}}{\alpha}, u_{*}-u_{\alpha}\right\rangle \\
+ & \gamma_{*}\left\langle h^{\prime}\left(u_{*}\right), \pi_{\overline{R\left(A^{*}\right)}}\left(u_{*}-u_{\alpha}\right)\right\rangle \\
& -\frac{\lambda_{\alpha}}{\alpha}\left\langle h^{\prime}\left(u_{\alpha}\right), \pi \frac{\pi_{\overline{R\left(A^{*}\right)}}}{\alpha}\left(u_{*}-u_{\alpha}\right)\right\rangle .
\end{aligned}
$$

Now, adding and subtracting $\left(\lambda_{\alpha} / \alpha\right)\left\langle h^{\prime}\left(u_{*}\right), \pi_{\overline{R\left(A^{*}\right)}}\left(u_{*}-u_{\alpha}\right)\right\rangle$ on the right side of this equality, it becomes

$$
\begin{aligned}
\left\|u_{*}-u_{\alpha}\right\| & \\
= & \left\langle\pi_{\overline{R\left(A^{*}\right)}}\left(u_{*}-u_{\alpha}\right)+\left(I-\pi_{\overline{R\left(A^{*}\right)}}\right)\right. \\
& \left.\times\left(A_{\eta}^{*}-A^{*}\right) \frac{A_{\eta} u_{\alpha}-f_{\delta}}{\alpha}, u_{*}-u_{\alpha}\right\rangle \\
+ & \left(\gamma_{*}-\frac{\lambda_{\alpha}}{\alpha}\right)\left\langle h^{\prime}\left(u_{*}\right), \pi_{\overline{R\left(A^{*}\right)}}\left(u_{*}-u_{\alpha}\right)\right\rangle \\
+ & \frac{\lambda_{\alpha}}{\alpha}\left\langle h^{\prime}\left(u_{*}\right)-h^{\prime}\left(u_{\alpha}\right), \pi_{\overline{R\left(A^{*}\right)}}\left(u_{*}-u_{\alpha}\right)\right\rangle .
\end{aligned}
$$

Observe that in case of $\left(I-\pi_{\overline{R\left(A^{*}\right)}}\right) h^{\prime}\left(u_{*}\right)=0$, we can take $\gamma_{*}=0$. Then, $0 \leq\left\langle h^{\prime}\left(u_{*}\right), u_{*}-u_{\alpha}\right\rangle=\left\langle h^{\prime}\left(u_{*}\right), \pi_{\overline{R\left(A^{*}\right)}}\left(u_{*}-\right.\right.$ $\left.\left.u_{\alpha}\right)\right\rangle$. From here and (78), we obtain

$$
\begin{aligned}
\| u_{*}- & u_{\alpha} \|^{2} \\
\leq & \left\langle\pi \frac{}{R\left(A^{*}\right)}\left(u_{*}-u_{\alpha}\right)+\left(I-\pi_{\overline{R\left(A^{*}\right)}}\right)\right. \\
& \left.\times\left(A_{\eta}^{*}-A^{*}\right) \frac{A_{\eta} u_{\alpha}-f_{\delta}}{\alpha}, u_{*}-u_{\alpha}\right\rangle \\
+ & \frac{\lambda_{\alpha}}{\alpha}\left\langle h^{\prime}\left(u_{*}\right)-h^{\prime}\left(u_{\alpha}\right), \pi_{\overline{R\left(A^{*}\right)}}\left(u_{*}-u_{\alpha}\right)\right\rangle .
\end{aligned}
$$

Finally, from here, by Lemmas 9 and 10, Theorem 8, and conditions (12) and (3), the estimate (74) follows.

In case of $\left(I-\pi_{\overline{R\left(A^{*}\right)}}\right) h^{\prime}\left(u_{*}\right) \neq 0$, the estimate (74) is an immediate consequence of Lemmas 7 and 9 , Theorem 8 , and conditions (12).

\section{Acknowledgments}

The authors would like to thank the reviewers for their valuable suggestions and comments. 


\section{References}

[1] W. H. Engl, M. Hanke, and A. Neubauer, Regularization of Inverse Problems, Kluwer Academic Publishers, Dordrecht, Netherlands, 1996.

[2] A. B. Bakushinsky and M. Y. Kokurin, Iterative Methods for Approximate Solution of Inverse Problems, vol. 577 of Mathematics and Its Applications, Springer, Dordrecht, Netherlands, 2004.

[3] B. D. O. Anderson and J. B. Moore, Optimal Control, Linear Quadratic Methods, Prentice Hall, Englewood Cliffs, NJ, USA, 1990.

[4] V. A. Morozov, Methods for Solving Incorrectly Posed Problems, Springer, New York, NY, USA, 1984.

[5] G. M. Vaŭnikko and A. Yu. Veretennikov, Iterative Procedures in Ill-Posed Problems, Nauka, Moscow, Russia, 1986 (Russian).

[6] I. Krnich and M. M. Potapov, "Projective sourcewise representability of normal solutions to linear equations on convex sets," Computational Mathematics and Mathematical Physics, vol. 41, no. 9, pp. 1315-1323, 2001.

[7] M. Jaćimović and I. Krnić, "On regularization of linear operator equation on convex set," Mathematica Montisnigri, vol. 28, pp. 5-11, 1999.

[8] A. Neubauer, "Tikhonov-regularization of ill-posed linear operator equations on closed convex sets," Journal of Approximation Theory, vol. 53, no. 3, pp. 304-320, 1988.

[9] J. Flemming and B. Hofmann, "Convergence rates in constrained Tikhonov regularization: equivalence of projected source conditions and variational inequalities," Inverse Problems, vol. 27, no. 8, Article ID 085001, 2011. 


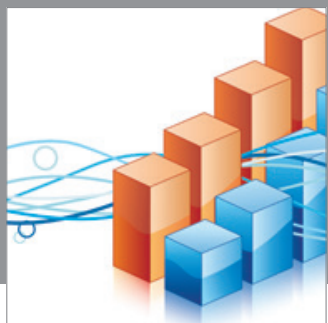

Advances in

Operations Research

mansans

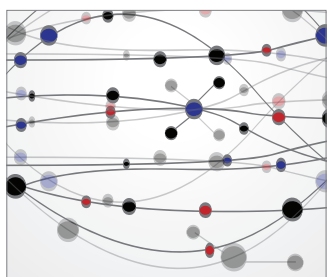

The Scientific World Journal
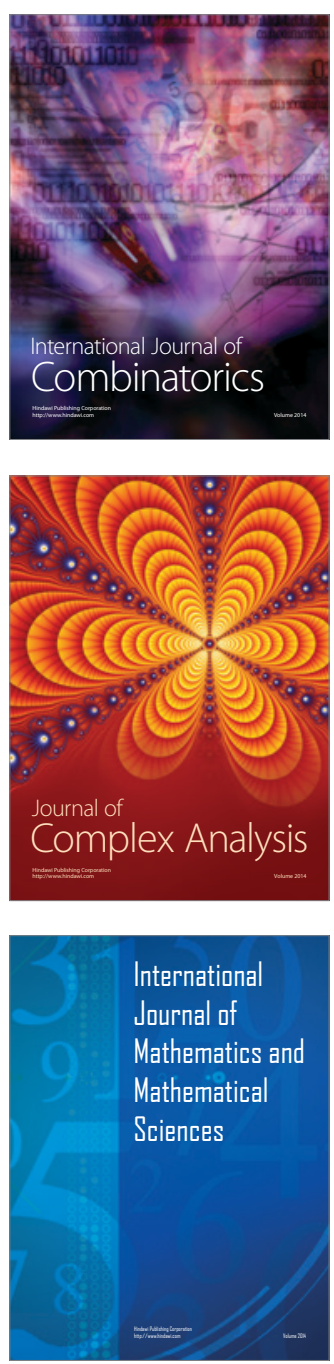
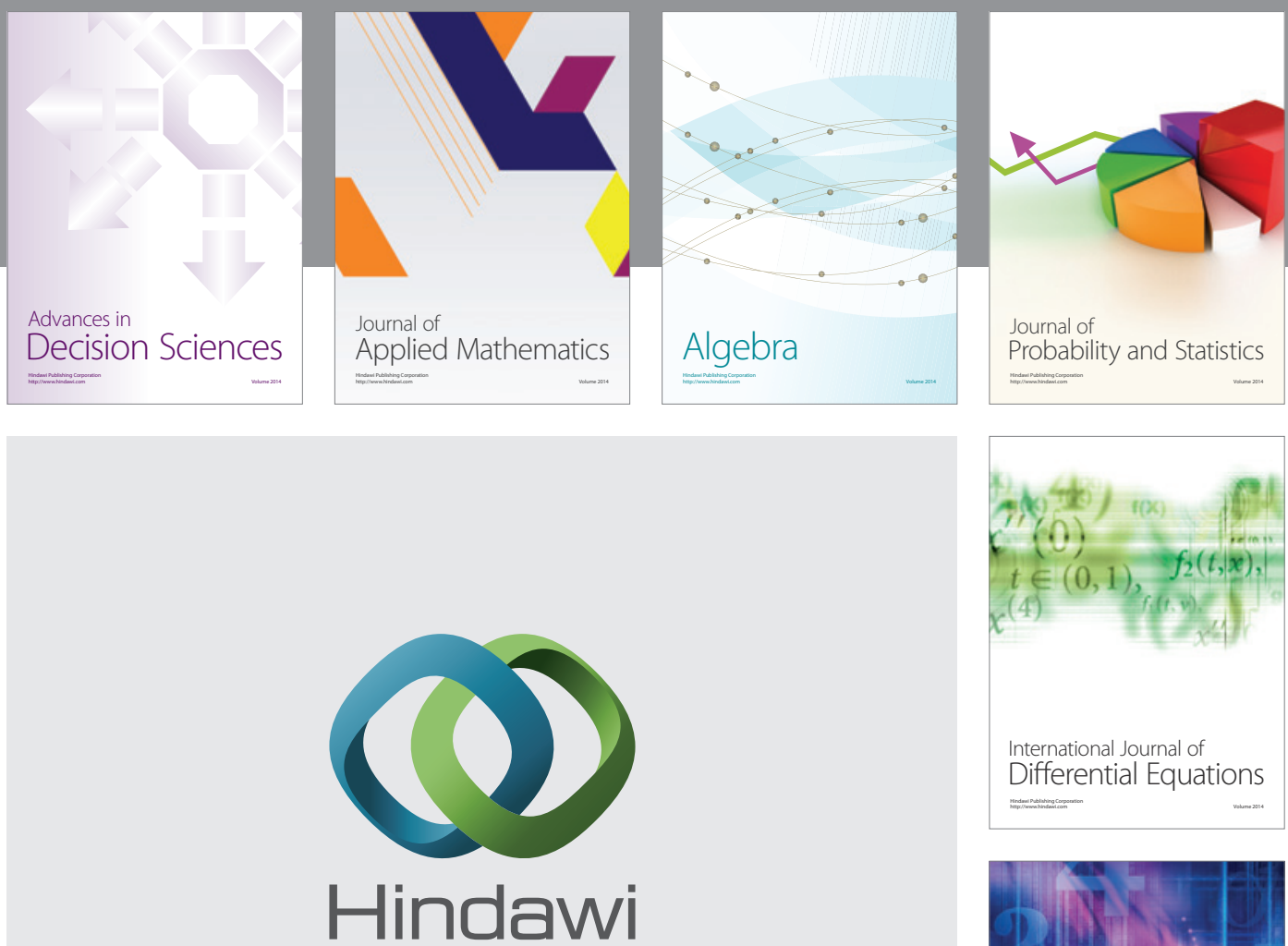

Submit your manuscripts at http://www.hindawi.com
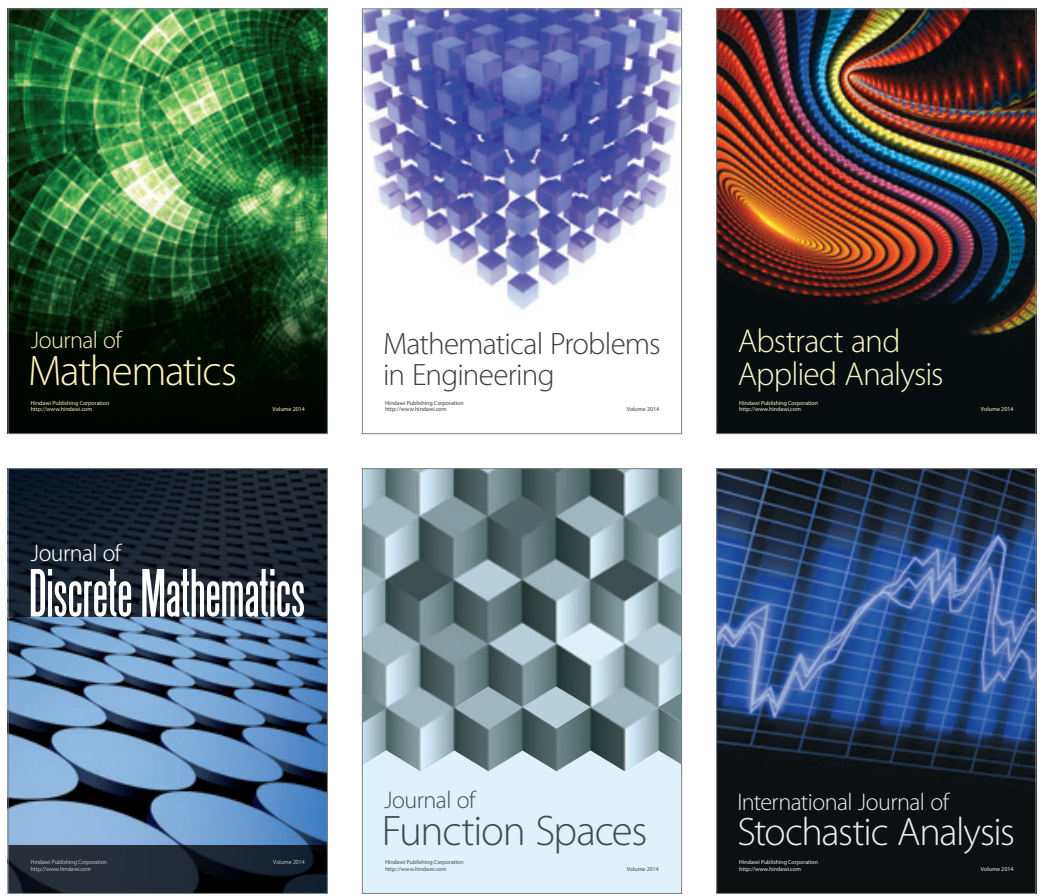

Journal of

Function Spaces

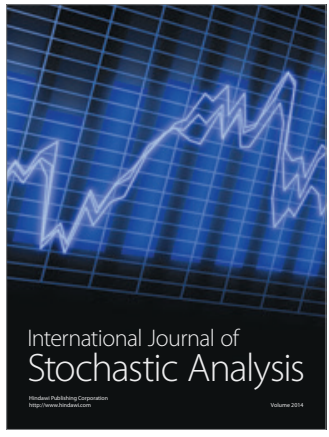

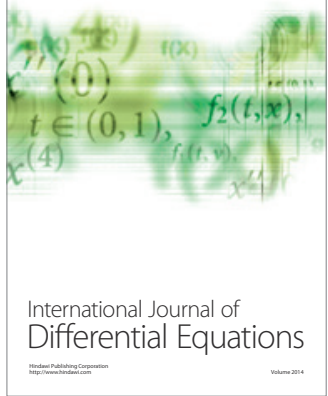
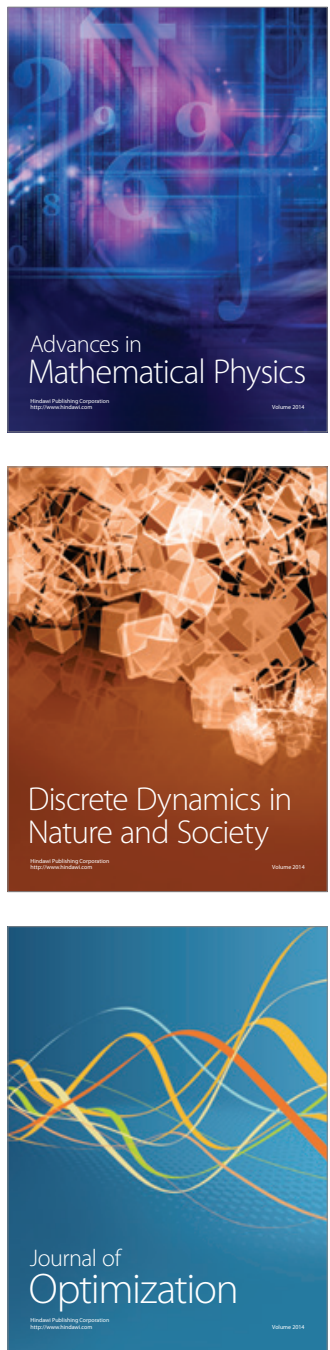